\title{
Assessment of Possibilities of On-Line Response Dynamic Traffic Management System Development in Medium Size Urban Areas
}

\author{
Drago SEVER, Robert LUTAR, Sebastian TOPLAK
}

\begin{abstract}
On-line response dynamic traffic management system (DTMS) seems to be the key solution for resolving many and the most important traffic problems in urban areas. Commonly used on-line response DTMS consists of on-road equipment (detectors, variable message signs etc.) and computer aided system. Such a system is generally installed in world metropolis with huge traffic loads that cannot be managed without it. The paper focuses on the real meaning of DTMS, possibilities of its usage in a medium size urban area, its purpose and expected consequences. Our experiment is based on the existing and verified traffic model of the City centre of Maribor, where different micro simulation scenarios were analysed. The main differences between non - response and on-line response traffic management systems are shown. In addition, their functionality and efficiency by resolving the possible traffic problem (unexpected traffic accident at the neutral point of the network) are represented.
\end{abstract}

Keywords: medium size urban area; simulation; traffic management

\section{INTRODUCTION}

In the last few decades, the increase of personal car flows is one of the biggest and the most growing problems in urban environment. The traffic (delays) has become the eliminator for development of the cities and their mobility. The range of the traffic flows exceeds the road and streets capacities in many cases and starts to devastate the quality of living conditions and natural environment especially in world metropolis. Many processes of suburbanization impact the growing dependence on personal cars which makes these problems bigger and bigger. Clearly, a traffic management system (TMS) is a well known solution to assure the efficient transportation system and in-time impact on the traffic flows in such conditions. The relevant equipment and tools for proactive traffic management and regulation (for example SCOOT, SCATS; AIMSUN ONLINE, Sitraffic- produced by Siemens A.G., Infrastructure \& Cities, Sector Mobility and Logistics Division; some modules already installed in Berlin, Braunschweig, Dubai, Essen and Prague [1]) already exist. In general, establishment of TMS is caused by problems in controlling traffic conditions in the cities and it impacts city investments.

Equally, growing dependence on a personal car is recognised in many Slovenian counties where over $85 \%$ of all daily trips were realised by personal cars in 2016 (the studies, prepared by UM FCTA are not published yet). The usage of public transport declined in the same period. The effect of mentioned conditions can be seen especially in peak hour in the city centres of main Slovenian cities (medium size urban area), where paths of different participants of urban traffic cross on the relative small area. In mentioned conditions, all unannounced extraordinary events such as traffic accidents or traffic infrastructure maintenance work could have important negative influence on the stage of city infrastructure - the result could be a significant growth of traffic delays, a negative impact on city living environment etc.

Taking into account that traffic will become the indicator of sustainable growth of the urban areas, the task of traffic engineers is to manage the city's traffic systems in such a way that the measures over time will guarantee efficient and equal mobility for all the participants. The mobility's aim is to guarantee fluid traffic flows without disturbance, almost without delays and with as little as possible unexpected events. It seems that traffic management systems (TMS) can also be used in medium size urban area which leads to a general question about expected advantages (benefits) if TMS is established and how to close the financial structure if the investment is reasonable.

\subsection{Research Problem, Goals and Research Hypothesis}

The city of Maribor is a typical European medium size city with about 100000 inhabitants. The old city centre (which is taken into consideration) is the location of almost all important city functions (municipality, courts of justice, health institutions, faculties, public transport facilities etc.). Almost all intersections in the analysed road network were signalised and coordinated from the main traffic centre situated in the Main bus station. The so-called nonresponse TMS based on coordinated signalised intersection is already established.

If city authorities want to develop a higher-level TMS, they have to deal with the relevant professional information about the expected advantages of a real traffic system in order to guarantee the quality of traffic flows and living conditions in the city. However, such a political decision should be based on assessments of the possibilities of different measures, which have to follow an analysis as realistically as possible and take into consideration local typical driver behaviour [2], too.

The only possible and the most efficient way to assess the efficiency of proposed TMS system is to use traffic simulation models. Based on TRB [3], traffic simulation models can represent real-time conditions such as delays, travel times, queues and flows. Models are divided on macro, mezzo and micro models, which depend on area, and details that are used.

The main goal of the research is to define a procedure to enable evaluation of establishment of different level TMS.

Proposed procedure is established and tested on the case of the road network in the centre of the city of Maribor 
using micro simulation model (experiment).

The research hypothesis: an on-line response dynamic traffic management system can significantly contribute to the traffic conditions in the road network of medium size urban areas.

\section{TRAFFIC SIMULATION MODEL}

Traffic simulation models have proven to be suitable tools for the evaluation of intelligent transport systems (ITS) such as advanced TMSs and adaptive traffic control systems. The applicability of traffic simulation mostly depends on computing performance. Off-line applications, such as those employed in testing ITS strategies and in planning studies, best operate with fast-running traffic simulation models because of their iterative nature. Models that are faster than real time are a requisite for on-line applications. They support decision-making in real-time traffic management. A key aspect in the evaluation of ITS strategies is the impact on drivers' behaviour, namely with respect to path choice. The dynamic traffic assignment (DTA) model emulates driver decision-making processes $[4,5]-$ Fig. 1 .
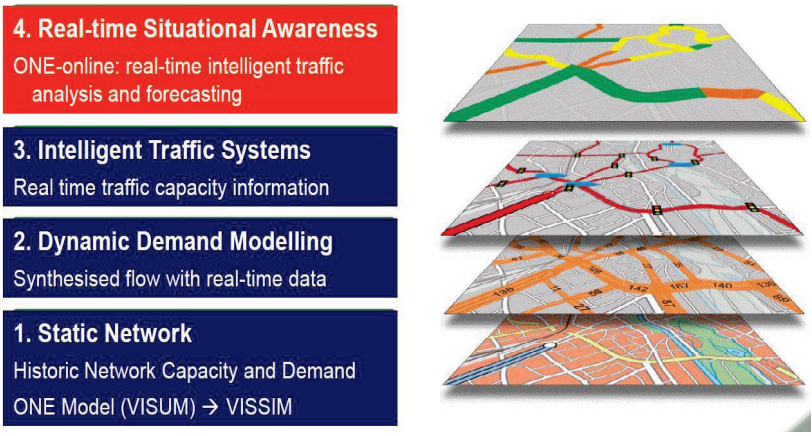

Figure 1 On-line modelling - Situational awareness [7]

The microscopic traffic simulation demonstrates the movement decisions of each vehicle inside a traffic network in every time step - typically every fraction of a second. These models are capable of modelling complex interactions between public and private vehicles, or between vehicles and pedestrians. Disaggregate nature of the models allows to realistically represent a variety of complex operational conditions although they require some effort to be put together [6].

A microsimulation traffic model allows for simulation of actual stage of activities on existing urban road network as well as simulation and analysis of different possible activities on it. Hence, the result seems to be realistic and depends on the quality of input data.

\section{EXPERIMENT: ON-LINE RESPONSE DYNAMIC TMS - CITY OF MARIBOR}

The evaluation of impact of establishing the on-line response dynamic TMS in real urban road traffic system was made using microsimulation traffic modelling. The relevant network and traffic data were captured; details are elaborated also by Kuhnt [8]. Although a great number of free and open-source software exists on the market such as the one elaborated by Rangel [9], application package Aimsun version 8.0.5, produced by TSS, Transport
Simulation System was used for simulation [10].

The procedure for assessment of possibilities of different levels in TMS development includes the following steps:

Step 1: establishment of a microsimulation model of a city road network (case: City of Maribor) based on real infrastructure, environmental and traffic data - also called Scenario 1 - Do Nothing (DN);

Step 2: presumption of a traffic accident in random position inside the existing road network;

Step 3: establishment of the conditions to simulate non-response TMS - also called Scenario 2 - Do Minimum (DM);

Step 4: establishment of the conditions to simulate online response of the dynamic TMS - also called Scenario 3 - Do Something (DS);

Step 5: comparison of the results of Step 1, 3 and 4 (results).

All mentioned steps were realised from the same infrastructural, environmental and traffic data and they are explained below. The selected time period was the time of afternoon peak hour between 15.00 and 16.00.

Step 1: Establishment of a microsimulation model of a city road network (case: City of Maribor) based on real infrastructure, environmental and traffic data Scenario 1 - Do Nothing (DN);

The microsimulation traffic model was prepared using the real infrastructure, environmental and traffic data, which were collected in the frame of the real existing traffic-planning project [11].

As a rule, micro simulation models use a realistic and geometrically accurate representation of the road network that includes the detailed specification of lanes, junctions, permitted turning movements, traffic signals, priorities and gradients. Traffic signals are reflected accurately, exhibiting fixed time, vehicle actuated and other control methods as required.

Vehicles were considered individually with properties such as vehicle size, acceleration rates, maximum speed, deceleration and breaking rates, speed limit acceptance and car following distance, to name a few. These parameters were sampled from probability distribution defined by the user as a part of the calibration process and therefore each vehicle had a unique set of parameters that govern its movement through the network. Using these parameters, and other parameters defined for the network, the vehicles were moved through the network following a number of behavioural models including car following, lane changing, gap acceptance, overtaking, merging and diverging models - details were also reported in Bradshaw and Stevenson [5] and Cvitanić [12].

Selected urban road traffic network represents the part of macro traffic model of the urban region of the City of Maribor (Fig. 2) and it includes:

- 629 road connections - links in total length of $32 \mathrm{~km}$,

- 119 intersections, 15 of them are equipped by Traffic Light Systems (TLS),

- 94 centroids, 74 internal and 16 external zones,

- OD matrix of afternoon peak hour between 15.00 and 16.00 including personal vehicles and public passenger transport,

- lines of public passenger transport; 
- $\quad$ additional control equipment (Fig. 3):

- 9 detectors for measurement of type of vehicle, traffic flows, speed, density, time space etc.,

- 5 variable message signs for driver's information.

The network was equal for all scenarios of the experiment.

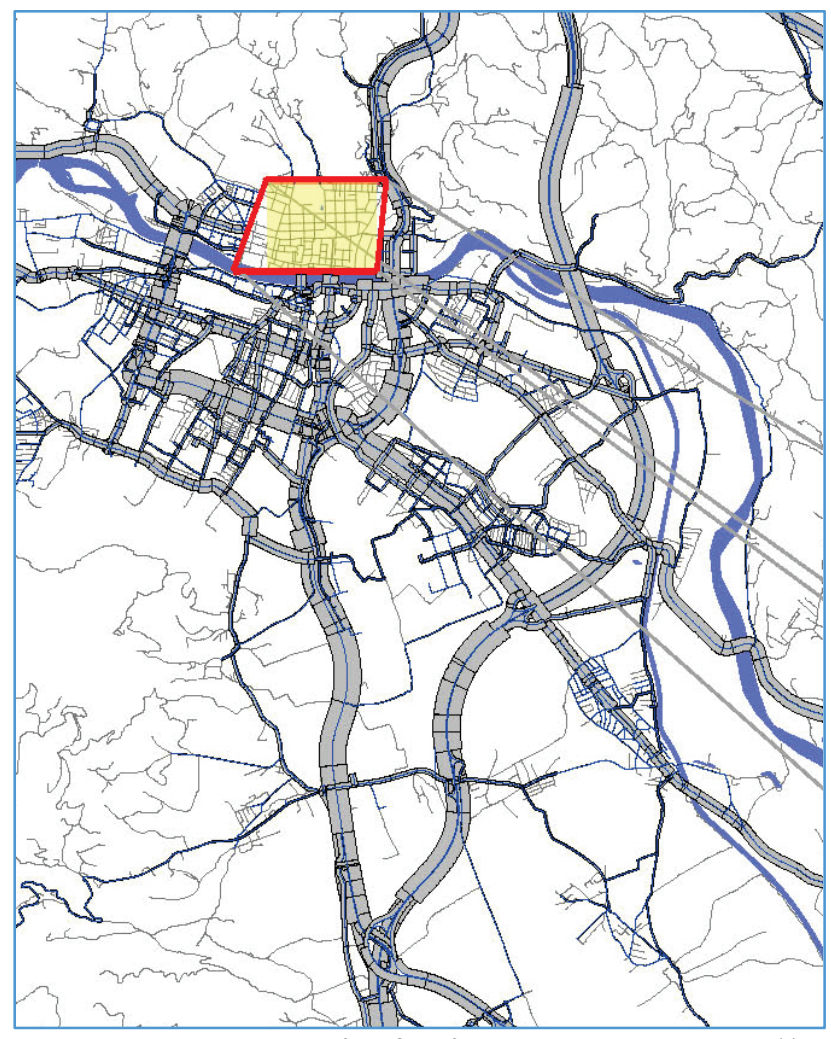

Figure 2 Macro urban region of the City of Maribor and selected network [11]

Step 2: Car accident happens

In the location signed as D1 in Fig. 3 at the time $t_{1}+$ $10 \mathrm{~min}$, a traffic accident happened. Due to regular intervention, all obstacles were removed at the time $t_{1}+10$ $\min +30 \mathrm{~min}$.

Characteristics of dynamic traffic simulation were:

- Simulation begins at $t_{1}=15.00$ and ends at $t_{2}=16.00$, simulation period of $60 \mathrm{~min}$,

TLS, C $=120 \mathrm{~s}$,

- Loading: Stochastic Route Choice,

- Two-Lane Car-Following model,

- Vehicle input: Exponential.

Step 3: Establishment of the conditions to simulate non-response TMS - Scenario 2 - Do Minimum (DM)

The do minimum scenario is a microscopic traffic simulation model, which was analysed by the predisposition of existing specific barrier (traffic accident). The simulation was equal to DN scenario, however, a collision was simulated 10 minutes after the start. The obstacles were there for 30 minutes. The location of the collision is shown in Fig. 3. Because of collision, one of the two possible lanes was closed and the speed was reduced to $5 \mathrm{~km} / \mathrm{h}$. This was applied for the mentioned section of the road only.

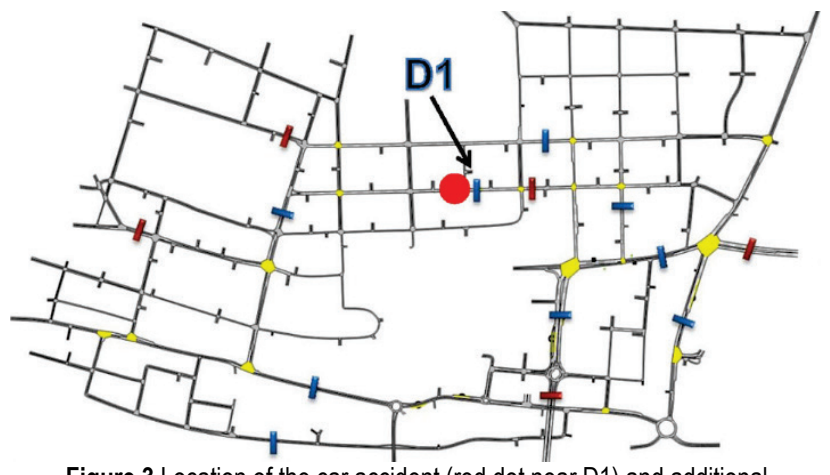

Figure 3 Location of the car accident (red dot near D1) and additional equipment [13]

Step 4: Establishment of the conditions to simulate on-line response of the dynamic TMS - Scenario 3 - Do Something (DS)

Do something scenario is a dynamic traffic microsimulation model with an on-line quick responsible TMS. Traffic management in this case depended on Strategies and Policies for its operations. A strategy is a collection (set) of policies to be applied to solve a problem, whereas a policy contains a collection of actions that are applied at the same time. An action is a modification of the traffic conditions or driver behaviour.

In DS scenario, some of possible actions were used with additional impact of pedestrians in selected intersection. In fact, three different measures were shown in the variable message signs (VMS) to inform the drivers (Fig. 4):

1) Rerouting vehicles out of the avenue (action Force turning),

2) Rerouting vehicles through a parallel street (action Force turning) and

3) Assign an alternative route (action Rerouting).

In order to get real field information about problems in traffic flow, detectors must be installed on roads and streets. They are necessary to activate triggers, which are used to activate policies automatically depending on several conditions.

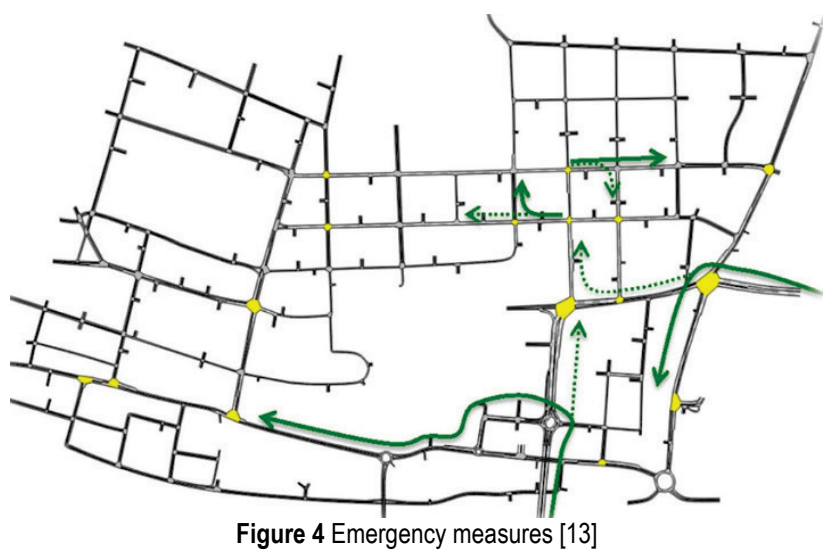

Description of emergency measures:

The first trigger will be activated at the same time as the first detector (location: on the same arterial as the accident) detects occupancy more than $80 \%$. The second trigger also relates to the same detector and is activated when occupancy falls under $50 \%$. In fact, triggers must be joined to the policy that will activate or deactivate 
emergency measures in the VMS.

\section{Step 5: Results comparison}

Comparison of results of Step 1, 3 and 4 are presented in Chapter 4.

\section{RESULTS}

To understand the consequences and differences between all three scenarios DN, DM and DS, the results of experiment are divided into two main parts. The first part includes the whole road network analysing all three scenarios, while the second part focuses on a section of the arterial, where the accident took place, analysing only DM and DS scenarios.

\subsection{Whole Road Network}

The summary results of experimental network in peak hour period are presented in Fig. 5 and Tab. 1. Fig. 5 shows the summary results for all scenarios; y axe represents the different units, which are listed under the analysed characteristics. In Tab. 1, only comparison between S2 and $\mathrm{S} 3$ is analysed in details.

To sum up, the usage of on-line response TMS - DS on analysed network under analysed conditions guarantees better traffic conditions parameters such as in the case of S2:

- $\quad$ average delays decreased by $14.37 \%$,

- $\quad$ average queue length decreased by almost $14 \%$ and total travel time decreased by almost $5 \%$.

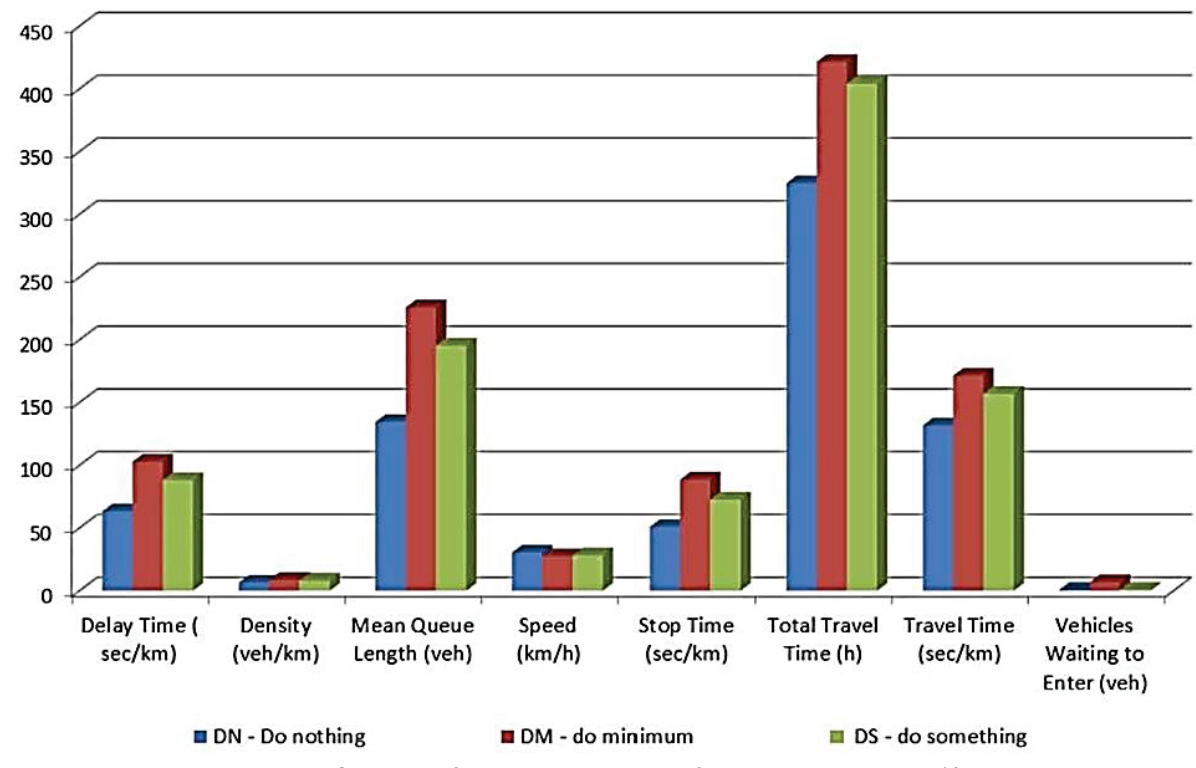

Figure 5 Summary of the experiment results for whole road network [13]

Table 1 Network Summary [13]

\begin{tabular}{|c|c|c|c|c|c|c|}
\hline \multirow{2}{*}{$\begin{array}{c}\text { Microscopic simulation based on } \\
\text { dynamical loading }\end{array}$} & \multicolumn{2}{|c|}{$\mathrm{S} 2-\mathrm{DM}$} & \multicolumn{2}{|c|}{ S3 - DS } & \multirow{2}{*}{\multicolumn{2}{|c|}{$\begin{array}{c}\mathrm{DS} / \mathrm{DM} \\
(\%)\end{array}$}} \\
\hline & Total & Stand. deviation & Total & Stand. deviation & & \\
\hline Delay Time $(\mathrm{s} / \mathrm{km})$ & 102.97 & 124.70 & 88.17 & 94.27 & -14.37 & $\mathrm{DS}<\mathrm{DM}$ \\
\hline Density (veh/km) & 8.77 & - & 8.16 & - & -6.96 & $\mathrm{DS}<\mathrm{DM}$ \\
\hline Flow (veh/h) & 7417 & - & 7582 & - & 2.22 & $\mathrm{DS}>\mathrm{DM}$ \\
\hline Mean Queue Length (veh) & 225.94 & - & 195.47 & - & -13.49 & $\mathrm{DS}<\mathrm{DM}$ \\
\hline Speed $(\mathrm{km} / \mathrm{h})$ & 27.85 & 11.76 & 28.32 & 11.36 & 2.42 & $\mathrm{DS}>\mathrm{DM}$ \\
\hline Stop Time $(\mathrm{s} / \mathrm{km})$ & 88.68 & 120.31 & 72.81 & 91.02 & -17.90 & $\mathrm{DS}<\mathrm{DM}$ \\
\hline Total Distance Travelled (km) & 5817.75 & - & 8840.29 & - & 51.95 & $\mathrm{DS}>\mathrm{DM}$ \\
\hline Total Travel Time $(\mathrm{h})$ & 421.55 & - & 404.25 & - & -4.10 & $\mathrm{DS}<\mathrm{DM}$ \\
\hline Travel Time (s/veh) & 171.57 & 124.44 & 157.06 & 94.18 & -8.46 & $\mathrm{DS}<\mathrm{DM}$ \\
\hline Vehicle Waiting to Enter & 7 & - & 1 & - & -85.71 & $\mathrm{DS}<\mathrm{DM}$ \\
\hline
\end{tabular}

Fig. 6 shows the analysis of the mentioned traffic parameters at the $10 \mathrm{~min}$ interval. It is easy to recognise the on line response of the TMS (DS scenario) - red line enables and guarantees that average delays and average density could decrease and average travel speed could increase on the analysed road network if traffic accident happens as it was represented.

\subsection{Section No. 20211}

The distinction of using different levels of TMS can be seen more thoroughly by observing just the section No. 20211, where the accident happened. Fig. 7 shows the changings in traffic flow parameters in 10 minute intervals of the analysed road section.

After the accident happened (15:10), delays increased in both scenarios, differences of $10 \%$ were recognised at 15:30 and 15:40 (20 minutes after the accident happened) - Fig. 7.

More differences are recognised by analysing the maximal queue length (veh) and the total stop time (s) on analysed section. The selected measures of on-line response DTMS - red line -enable almost normal traffic flow on the section after 1 hour, and the total (loss) time of stopped vehicle is almost $50 \%$ lower. 

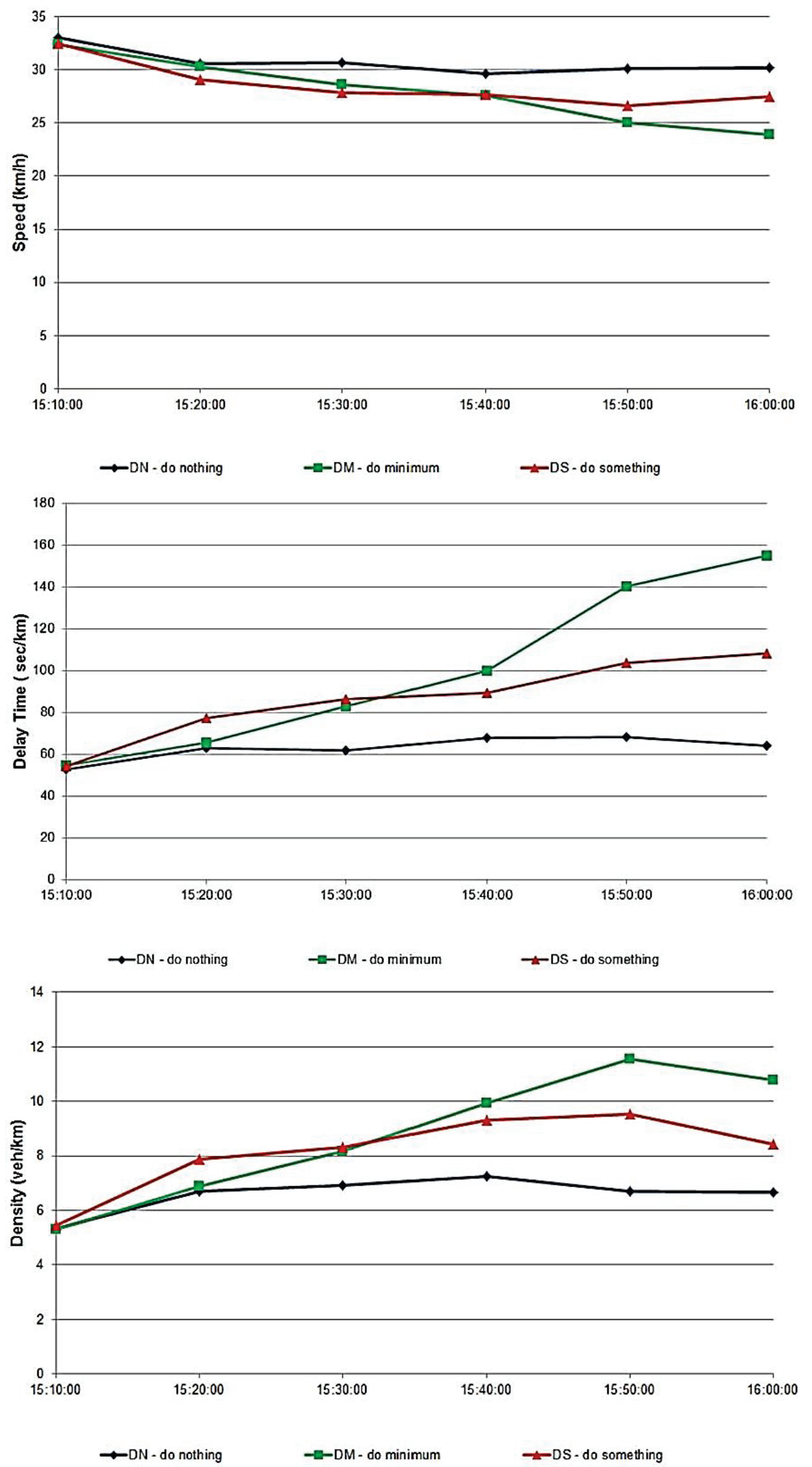

Figure 6 Average delays, average density and speed on whole network - 10 min intervals, accident happened at 15:10 [13] 

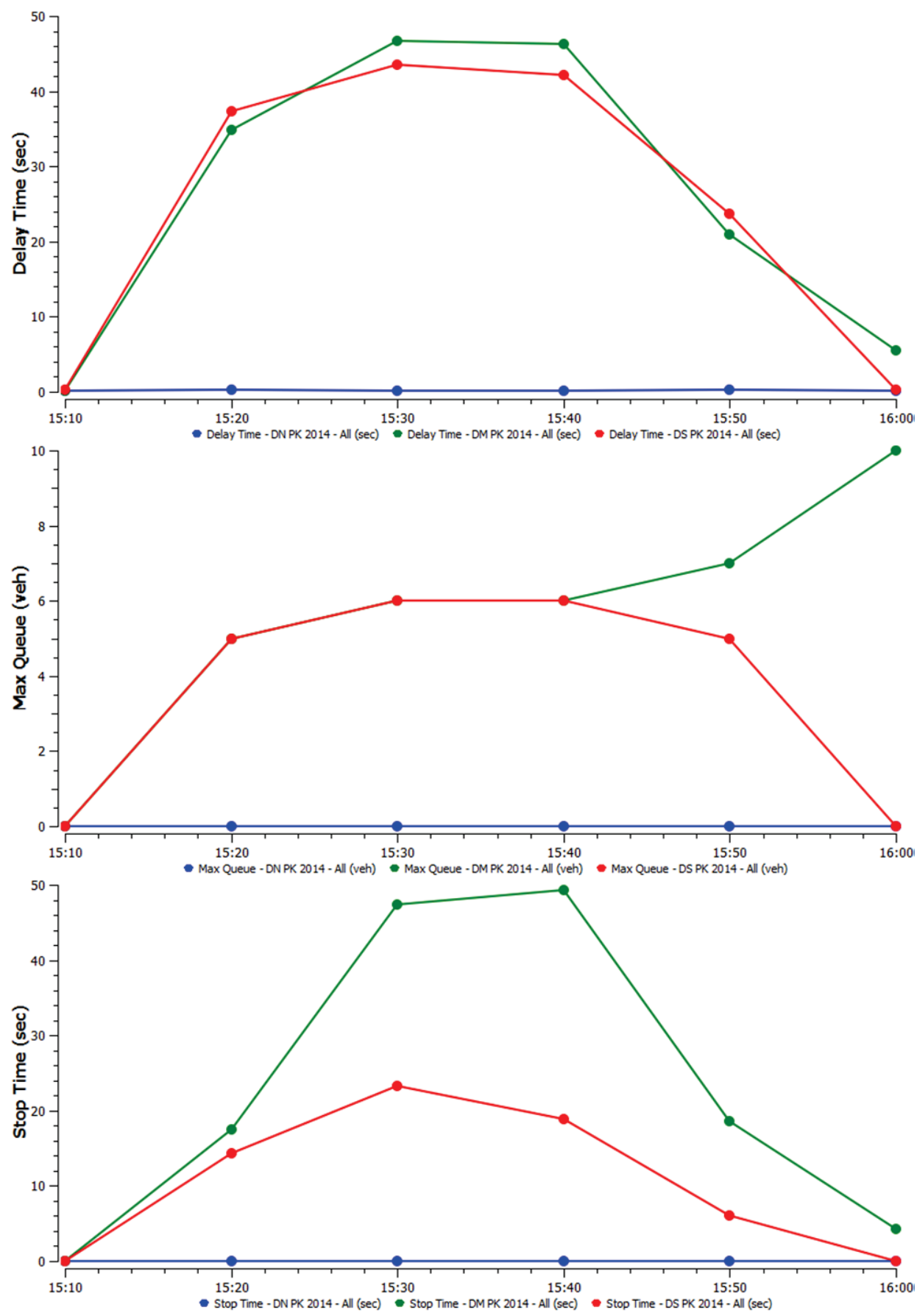

Figure 7 Average delays (s) on section No. 20211 during 10 min intervals of analysed peak hour [14]

\section{CONCLUSIONS}

The research work in the paper has discussed the procedure of assessing the possibilities of the TMS establishment in a medium size city area. The procedure consists of five successive steps representing different levels of TMS. Based on the results, the research hypothesis is confirmed.

Using the procedure of three different scenarios with intention to investigate the meaning and possible consequences of establishment of on-line response, the TMS in the selected urban area (the city centre of Maribor) is presented. Our research reveals that on the medium-size road network DS scenario can handle a specific barrier, if the network, detection and driver information work with hand in hand in real time.

The experiment also shows many advantages of the
TMS response and it is from the traffic engineering point of view absolutely better choice to implement. Such professional proof could contribute to better and easier political decisions. However, a more detailed research of acceptability should also consider an economic and environmental aspect.

Finally, it should be mentioned that the establishment of on-line TMS response is besides the cost of computer software and hardware linked with relatively high costs of equipment (detection and Variable message sights) which increase with the scale of urban road traffic network.

\section{REFERENCES}

[1] Siemens AG. (2012). Sitraffic Concert, Sitraffic Scala and Sitraffic Guide: Three complex tasks - one common platform. München, Germany. 
[2] Bošnjak, I., Jusufranić, I., \& Višnjić, V. (2004). Modeling framework for dynamic multiclass traffic assignment in ITS environment. Promet - Traffic \& Transportation, 16(2), 7176.

[3] TRB. (2009). Traffic Flow Theory, Characteristics, and Simulation Models. TRR 2124, TRB.

[4] Bradshaw, A. \& Stevenson, P. (2011). Microsimulation: an important tool in the designer's armoury. TEC Magazine, $52(10), 431-436$.

[5] Meng, M., Shao, C., \& Zeng, J. (2014). A simulation-based dynamic traffic assignment model with combined modes. Promet - Traffic \& Transportation, 26(1), 65-73. https://doi.org/10.7307/ptt.v26i1.1252

[6] Papayannoulis, V., Scilaci, S., \& Torday, A. (2011). True grid. Traffic Technology International, Oct./ Nov., 91-92.

[7] Robinson, J. \& Jackman, G. (2010). Traffic control and signal modelling in the Capital - A 2020 approach in 2010. Roads Summit, Sydney, Australia.

[8] Kuhnt, S. \& Wenzel, S. (2010). Information acquisition for modelling and simulation of logistics network. Journal of Simulation, 4(2), 109-115. https://doi.org/10.1057/jos.2009.9

[9] de Assis Rangel, J. J. \& Cordeiro, A. C. A. (2015). Free and Open-Source Software for Sustainable analysis in logistics system design. Journal of Simulation, 9(1), 27-42. https://doi.org/10.1057/jos.2014.17

[10] TSS-Transport Simulation Systems. (2011). Aimsun user's manual, v. 7. Spain.

[11] CPI. (2014). Area of old city centre of Maribor on the left side of the river Drava (in Slovene). Project for Municipality of Maribor.

[12] Cvitanić, D., Breški, D., \& Lovrić, I. (2012). Possibility of micro-simulation models calibration - case study of the city of Split. Promet - Traffic \& Transportation, 24(3), 231-241.

[13] Lutar, R. (2015). On-line response dynamic traffic management system in urban areas. M.Sc. thesis (in Slovene). Faculty of Civil Engineering, Transportation Engineering and Architecture, University of Maribor.

\section{Contact information:}

Drago SEVER, Ph.D.

University of Maribor,

Faculty of Civil, Transportation Engineering and Architecture,

Smetanova 17, 2000 Maribor, Slovenia

drago.sever@um.si

Correspondention author

\section{Robert LUTAR, M.Sc.}

Nigrad d.d.

Zagrebška 30, 2000 Maribor, Slovenia

robert.lutar@nigrad.si

Sebastian TOPLAK, M.Sc.

University of Maribor,

Faculty of Civil, Transportation Engineering and Architecture,

Smetanova 17, 2000 Maribor, Slovenia

sebastian.toplak@um.si 